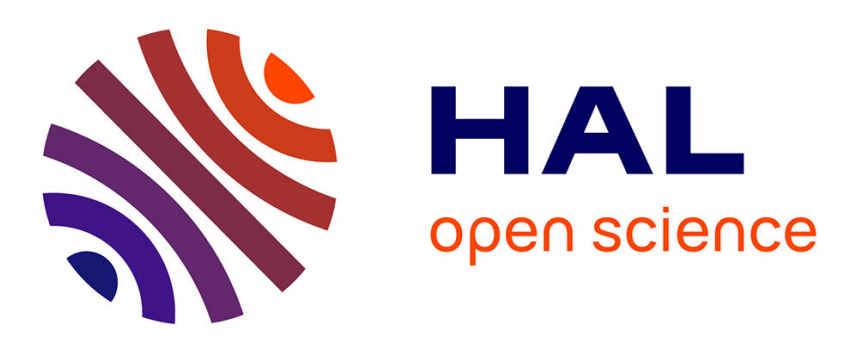

\title{
Influence of the calcaneus shape on the risk of posterior heel ulcer using 3D patient-specific biomechanical modeling.
}

V Luboz, A Perrier, M Bucki, B Diot, F Cannard, N Vuillerme, Yohan Payan

\section{- To cite this version:}

V Luboz, A Perrier, M Bucki, B Diot, F Cannard, et al.. Influence of the calcaneus shape on the risk of posterior heel ulcer using 3D patient-specific biomechanical modeling.. Annals of Biomedical Engineering, 2015, 43 (2), pp.325-35. 10.1007/s10439-014-1182-6 . hal-01083508

\section{HAL Id: hal-01083508 https://hal.science/hal-01083508}

Submitted on 17 Nov 2014

HAL is a multi-disciplinary open access archive for the deposit and dissemination of scientific research documents, whether they are published or not. The documents may come from teaching and research institutions in France or abroad, or from public or private research centers.
L'archive ouverte pluridisciplinaire HAL, est destinée au dépôt et à la diffusion de documents scientifiques de niveau recherche, publiés ou non, émanant des établissements d'enseignement et de recherche français ou étrangers, des laboratoires publics ou privés. 


\title{
Influence of the calcaneus shape on the risk of posterior heel ulcer using 3D patient-specific biomechanical modeling
}

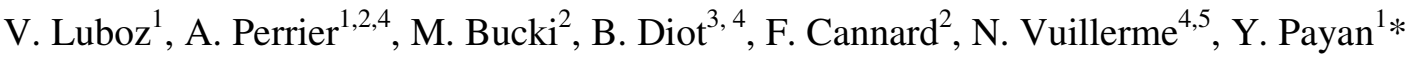 \\ 1 UJF-Grenoble1/CNRS/TIMC-IMAG UMR 5525, Grenoble, F-38041, France, \{vluboz, \\ aperrier, ypayan\} @imag.fr; \\ ${ }^{2}$ TexiSense , Montceau-les-Mines, France, \{marek.bucki, francis.cannard $\} @$ texisense.com; \\ ${ }^{3}$ IDS, Montceau-les-Mines, France, b.diot@ids-assistance.com; \\ ${ }^{4}$ UJF-Grenoble1/AGIM, Grenoble, F-38041, France, Nicolas.Vuillerme@ agim.eu \\ ${ }^{5}$ Institut Universitaire de France, Paris, France
}

Abbreviated title: Biomechanical model for heel ulcer prevention

*Corresponding author:

Yohan Payan

Equipe GMCAO - Laboratoire TIMC-IMAG

Université Joseph Fourier - CNRS UMR 5525

Pavillon Taillefer

Faculté de Médecine - 38706 La Tronche cedex - France

Tel: +33 (0)4 56520001 - Fax: +33 (0)4 56520055

email: Yohan.Payan@imag.fr

To appear in Annals of Biomedical Engineering 


\begin{abstract}
Most posterior heel ulcers are the consequence of inactivity and prolonged time lying down on the back. They appear when pressures applied on the heel create high internal strains and the soft tissues are compressed by the calcaneus. It is therefore important to monitor those strains to prevent heel pressure ulcers. Using a biomechanical lower leg model, we propose to estimate the influence of the patient-specific calcaneus shape on the strains within the foot and to determine if the risk of pressure ulceration is related to the variability of this shape. The biomechanical model is discretized using a 3D Finite Element mesh representing the soft tissues, separated into four domains implementing Neo Hookean materials with different elasticities: skin, fat, Achilles' tendon, and muscles. Bones are modelled as rigid bodies attached to the tissues. Simulations show that the shape of the calcaneus has an influence on the formation of pressure ulcers with a mean variation of the maximum strain over 6.0 percentage points over 18 distinct morphologies. Furthermore, the models confirm the influence of the cushion on which the leg is resting: a softer cushion leading to lower strains, it has less chances of creating a pressure ulcer. The methodology used for patient-specific strain estimation could be used for the prevention of heel ulcer when coupled with a pressure sensor.
\end{abstract}

Key terms: posterior heel ulcer prevention, biomechanical model, patient specific. 


\section{Introduction}

Two fifth of the patients being taken in charge by a reanimation or a geriatric unit will develop a pressure ulcer. $40 \%$ of those ulcers are located on the posterior side of the heel, mostly because patients stay for hours lying on their back without moving (Perneger et al. ${ }^{1}$ ). This condition is often followed by an amputation of part of the foot. In 2007, in the US alone, the cost of pressure ulcers was estimated to be 48 billion USD (Driver et al. ${ }^{2}$ ) and survival rate after 5 years was only $50 \%$ following a foot amputation $\left(\right.$ Reiber $\left.^{3}\right)$. The main factors of pressure ulcer development are the excessive intensity and the repetition of pressures applied on the foot. This is worsened when the patient suffers from diabetes with a neuropathy that reduces or even suppresses foot sensitivity. Three mechanisms, at least, are recognized as leading to pressure ulcerations $\left(\right.$ Mueller $^{4}$, Loerakker ${ }^{5}$ ): (1) ischemia caused by increased pressure duration, (2) high tissue strains created by increased pressure magnitude, and/or (3) tissue fatigue caused by increased number of pressure loads. Pressure ulcers created in reanimation or geriatric units mainly stem from the first two mechanisms.

Pressure ulcer prevention is mainly based on the patient's daily vigilance and on monitoring of the skin condition by the medical staff. When no obvious external signs are visible on the patient's skin, both the patient and the clinical staff tend to release their attention. Unfortunately, when the first actual symptoms appear, serious complications have already begun. Reducing the pressure load at the interface between the heel and the source of 
trauma prevents further ulceration and facilitates wound healing (Armstrong et al. ${ }^{6}$ ). This can be done with devices such as casts, orthotics, insoles or foam bandages for example.

To assist patient monitoring, devices measuring pressures at the interface between the bed and the body were introduced few years ago. For example, Hill-Rom (http://www.hillrom.com/) and DTH (http://www.dth.re/) proposed commercial devices comprising a mattress with several pneumatic actuators allowing control over the pressure patterns underneath the patient. Such medical beds can change the levels of pressure beneath the buttocks, the heels, or the legs thus avoiding sustained local overpressures. Unfortunately, their price and their fairly limited efficiency (due to a limited number of pneumatic actuators) limit their relevance in reanimation and geriatric units in the case of a long term daily prevention routine. Moreover, their actions are also limited because these pneumatic systems alternate high and low pressures in a cyclic pattern which is not always the best suited option for the patient. Indeed measuring the pressures below the patient's body to predict tissues' internal stresses and strains and adapting the pneumatic actuators accordingly would be more efficient. However internal stress and strain estimation is not possible with simple pressure sensors at the interface between the skin and the bed (Linder-Ganz et al. ${ }^{7}$ ). For example, a similar pressure distribution could be observed under the heel of a thin person with blunt calcaneus bone and a heavy person with sharp calcaneus bone even though the latter is probably more at risk. Indeed, as shown by Prof. Gefen ${ }^{8}$, the likelihood of the formation of a pressure ulcer depends on the calcaneus bone curvature as well as the thickness of the soft tissues underneath. In this article, Prof. Gefen used an analytical model to study the influence of the calcaneus' shape and the elasticity of the soft tissues on the internal deformations. Although 
the calcaneus was modeled as a simple sphere (Hertz analytic model) and the soft tissues as a flat volume, the study suggested that atypical foot anatomies (characterized by heavy-weight foot, sharp posterior calcaneus and thin soft tissue padding) are theoretically more prone to heel ulcers.

In order to take into account these anatomical differences and to quantitatively assess the internal stresses and strains from the measured external pressures, several studies have proposed (1) to build a patient-specific biomechanical model of the foot including soft tissues and bony prominences, and (2) to use this numerical model to compute the internal strains and stresses (in real-time wherever possible). Ledoux et al. ${ }^{9}$ modelled the soft tissues under the foot (skin, fat and muscles) as a Finite Element (FE) mesh with a homogeneous linear elastic material, the bones as another set of rigid FE meshes, the joints' actions as contacts between the bones, and the ligaments connecting the bones located in the mid foot as cables. Chen et al. ${ }^{10}$ proposed a more realistic version of an FE foot model including almost all the foot ligaments and using a large deformations framework with a Mooney Rivlin constitutive law for the soft tissues of the whole foot. Even though this model is fairly complete, it lacks computational efficiency and does not separate the different tissue types. These goals were partially addressed by the model of Luboz et al. ${ }^{11}$. In this work, the foot soft tissues are represented as four different Neo Hookean materials implementing respectively the plantar skin, the non-plantar skin, the fat, and the muscles. Bones are modeled as rigid bodies. Focusing on the heel, Sopher et al. ${ }^{12}$ used an FE model with different tissue layers to study the effects of two foot postures on different supports (simulating the bed supporting the heel).

Nevertheless, none of these works have studied the consequences of the variability in the shape/morphology of the calcaneus bone in terms of risks of pressure ulceration. The four 
models listed above were indeed based on the morphology of a single patient; it was therefore not possible to evaluate the effect of different calcaneus shapes on the internal strains (in the remainder of the article only the strains will be considered as this seems to be the current consensus in terms of pressure ulcer etiology, Loeraker et al. ${ }^{5}$ ). In addition, these models omit the calf in their simulation despite the known fact that this structure plays an important role on the pressure applied on the heel while lying down. The aim of the present work is therefore to quantify the influence of various calcaneus shapes on the risk of pressure ulceration. This risk is assessed by quantifying the internal strains and the amount of tissue volume involved in the deformation. To this end, (1) the biomechanical foot model presented in Luboz et al. ${ }^{11}$ was extended to include the calf as well as an FE model of a cushion composed of three compartments (under the heel, the Achilles tendon and the calf); and (2) calcaneus shapes collected from 18 patients (figure 1) were used to create 18 distinct FE models. These models were built from the same external foot geometry which means that only the calcaneus shape changes from one model to the other (figure 2). We therefore expect the simulated internal strains to vary below the heel from one subject to another while being almost constant below the calf, where all 18 subjects share the same simulated morphology.

\section{Materials and Methods}

Each biomechanical model described in this study was implemented on the 3D simulation platform, ArtiSynth ${ }^{13}$ (www.artisynth.org). 


\subsection{Heel model generation}

The geometry of the domain of the biomechanical model shared by all subjects (i.e. before the inclusion of each specific calcaneus bone) is based on the Zygote database (www.zygote.com). Surfaces from the lower leg's skin, muscles, and bones, as well as the Achilles tendon were extracted from this database. The calcaneus alone was left out to be replaced by each of the 18 bones in the database (figure 1). To make sure that the same amount of tissues were present between the calcaneus and the skin, all 18 calcanei were positioned so that their most posterior (lower) tips would be superimposed, figure 3 .

Based on these surfaces, an automatic FE mesh generator (Lobos et al. ${ }^{14}$ ) was used to generate a mesh of the muscles, fat and skin layers with a minimum of tetrahedral (to limit the locking effect observed in quasi-incompressible assumptions) while keeping a smooth and accurate boundary between the different structures using transition elements such as pyramids and wedges. This led to a set of 18 meshes having approximately 122,000 elements, including approximately 29,000 hexahedrons, 38,000 pyramids, 28,000 wedges, and 27,000 tetrahedrons, for an approximate total number of nodes of 66,000 . To keep the number of elements as low as possible while maintaining a high density in the region of interest, about a third of the leg soft tissues (above the tibia) was longitudinally removed, figure 4. This removal has no influence on the simulation results as the main deformations are recorded below the calf and the heel and the bony structures prevent them from spreading in the soft tissues above the leg bones. The mesh generator left holes in the soft tissue mesh to simulate the bones, implemented as rigid bodies. 
During the simulation, the leg lies on a cushion whose geometry was created in Blender (www.blender.org) to represent a typical pneumatic cushion used on geriatric beds (figure 4).

\subsection{Heel soft tissues materials}

The FE mesh has four layers of soft tissues: skin, fat, Achilles tendon, and muscles. They are modeled using four different compressive Neo Hookean materials (Bonnet \& Wood $^{15}$ ) with respective Young moduli set to $200 \mathrm{kPa}$ for the skin, $30 \mathrm{kPa}$ for the fat, $1 \mathrm{GPa}$ for the tendon, and $60 \mathrm{kPa}$ for the muscles, as shown in figure 4 . Such a material takes into account large deformations, is fairly stable at compressive strains and exhibits characteristics that can be identified with the familiar material parameters found in linear elastic analysis. Assuming these tissues are quasi-incompressible, we set their Poisson ratio to 0.495 , except for the fat for which a value of 0.49 is used. These values were proposed by Sopher et al. ${ }^{12}$.

A single $1 \mathrm{~mm}$ thick layer of elements is used to simulate the skin. It completely surrounds the leg except on the tibial anterior and proximal knee clip planes. The shapes of both the muscle volume and the Achilles tendon were defined from the surface of the Zygote database. In the current modelling approach, no ligaments were modeled except for the Achilles tendon which is assumed to have a major influence in the occurrence of posterior

heel pressure ulcer (Cheung et al. ${ }^{16}$ ). The fat volume was defined as the domain between the muscle/bone/tendon domain and the inner skin layer. The elements in each distinct domain were assigned the corresponding elastic parameters, figure 4. 
The cushion was discretized with finite elements using the same automatic mesher. It is composed of 5,841 elements including 5,251 hexahedrons and 590 wedges respectively, for a total of 7,080 nodes. It is modelled using a Neo Hookean material of varying Young's moduli, to simulate the different pressures inside the cushion, and with a Poisson ratio of 0.495 to simulate quasi-incompressibility. The Young's moduli chosen for the cushion are chosen to produce a pressure at the interface between the leg and the cushion ranging between 1 and $8 \mathrm{~N} . \mathrm{cm}^{-2}$ in order to reproduce interface pressure measurements acquired with a Zebris pressure sensor (www.zebris.de), for a $70 \mathrm{~kg}$ young healthy subject in supine position. It leads to Young moduli varying from $10 \mathrm{kPa}$ to $500 \mathrm{kPa}$. The cushion is split into three sections of equal lengths corresponding to the calf, the Achilles tendon, and the back of the heel. The Young moduli of these sections can be set independently to simulate different pressure patterns below each one of these three sections of the leg.

\subsection{Boundary conditions}

To model the interactions between the leg and the cushion, the leg is dropped from 1 $\mathrm{mm}$ above the cushion and is subject to gravity. The leg's motion is controlled by simulated hip and knee joints, both modelled as cylindrical joints allowing rotation around one axis. The surface FE nodes on the tibia, fibula, foot bones as well as those lying on the anterior and proximal clip planes are rigidly linked to these leg bones to model soft tissues attachments. No sliding between the soft tissues and the bones is assumed. Overall, the bones (femur not included) and the soft tissues weight $4.2 \mathrm{~kg}$ in our simulation (assuming an individual 
weighing $70 \mathrm{~kg}$ and based on body proportions defined by Harless ${ }^{17}$ who reported that the lower leg and foot weigh $6 \%$ of the individual's total weight).

The simulation ends when the leg has stopped moving and rests in equilibrium on the cushion. Five simulations are performed for each of the 18 calcaneus shapes: (1) with the cushion completely soft (i.e. with a pressure interface between the leg and the cushion of approximately $1 \mathrm{~N} \cdot \mathrm{cm}^{-2}$ ), (2) with the section below the calf mildly inflated but the rest of the cushion soft (i.e. with an interface pressure between the heel and the cushion around $1 \mathrm{~N} . \mathrm{cm}^{-2}$ and between the calf and the cushion around $1.5 \mathrm{~N} . \mathrm{cm}^{-2}$ ), (3) with the section below the heel mildly inflated but the rest of the cushion soft (i.e. with an interface pressure between the heel and the cushion around $3.5 \mathrm{~N} . \mathrm{cm}^{-2}$ and between the calf and the cushion around $1 \mathrm{~N} . \mathrm{cm}^{-2}$ ), (4) with all the section mildly inflated (i.e. with an interface pressure between the heel and the cushion around $2 \mathrm{~N} . \mathrm{cm}^{-2}$ and between the calf and the cushion around $1 \mathrm{~N} . \mathrm{cm}^{-2}$ ), and finally (5) with the section below the heel highly inflated but the rest of the cushion soft (i.e. with an interface pressure between the heel and the cushion around $8 \mathrm{~N} . \mathrm{cm}^{-2}$ and between the calf and the cushion around 1 N.cm ${ }^{-2}$ ).

\section{Results}

Each simulation takes about 70 minutes on a PC with an INTEL CORE QUADRO i7 at $3.4 \mathrm{GHz}$ and 8 Go of RAM.

Given that pressure ulcers mostly result from high internal strains, the maximum Von Mises strain is monitored to assess the level of soft tissue compression in the model during each simulation. This criterion was also used in previous studies (Linder-Ganz et al. ${ }^{7}$ ) 
(Oomens et al. ${ }^{18}$ ) and is corroborated by the work of Loerakker et al. ${ }^{5}$ stating that prolonged pressures leading to strains above $20 \%$ in the soft tissues for more than two hours can lead to pressure ulcers. Furthermore, this work ${ }^{5}$ showed that even compressions lasting around ten minutes can lead to ulcers if they induce tissue strains above $50 \%$. These $20 \%$ and $50 \%$ Von Mises (VM) strain thresholds were consequently monitored in our simulations. In addition to that, the volume of the largest zone with contiguous nodes with VM strains above 20 and 50 $\%$ are monitored to observe the size of the region where pressure ulcers potentially occur. These regions are referred to as "clusters" in the rest of the paper. This paradigm is less subject to numerical instabilities than the maximal VM strain value alone and the associated volume gives a better idea of the level of tissue suffering in each simulated situation.

Table 1 summarizes the results obtained for all the simulations. For each of the five simulated cushions, the table gives the average on all 18 subjects of the volumes of the largest cluster with a VM strain above $20 \%$, the volume of the largest cluster with a VM strain above $50 \%$, and the average of the maximum VM strains in these clusters. For these data, table 1 also provides the standard deviation in $\mathrm{mm}^{3}$ and in $\%$ and the deviation in percentage points. It is consequently possible to see that in the first case (i.e. soft cushion with a pressure

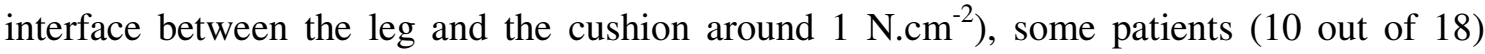
barely pass the $20 \%$ VM strain threshold limit and a mean cluster volume of $33 \mathrm{~mm}^{3}$ can then be observed. For a cushion with one or more of its sections mildly inflated (case 2, 3 and 4), the VM strain threshold limit of $20 \%$ is reached for all 18 patients. We observe a cluster of $223 \mathrm{~mm}^{3}$ and a maximum VM strain of $23.9 \%$ in case 2 , when the section below the calf is mildly inflated while the rest of the cushion remains soft (i.e. with an interface pressure between the heel and the cushion around $1 \mathrm{~N} \cdot \mathrm{cm}^{-2}$ and between the calf and the cushion 
around $1.5 \mathrm{~N} . \mathrm{cm}^{-2}$ ). The standard deviations for both the cluster volume and the VM strain are close to null, showing that the differences in the calcaneus bone shapes do not influence the risk of heel pressure ulcer creation when the cushion is more inflated under the calf (case 2 in table 1). When the section below the heel is mildly inflated while the rest of the cushion is soft (i.e. with an interface pressure between the heel and the cushion around $3.5 \mathrm{~N} . c m^{-2}$ and between the calf and the cushion around $1 \mathrm{~N} \cdot \mathrm{cm}^{-2}$ ), a cluster of $2,698 \mathrm{~mm}^{3}$ and a maximum VM strain of $34.8 \%$ are observed. The standard deviation for the cluster volume is $327 \mathrm{~mm}^{3}$ (12.1 percentage points (ppt)) while it is $3.6 \%$ for the VM strain $(10.4 \mathrm{ppt})$, showing that the differences in the calcaneus bone shapes do influence the risk of pressure ulcer creation when the cushion is more inflated under the heel (case 3 in table 1). When the cushion is uniformly mildly inflated (i.e. with an interface pressure between the heel and the cushion around 2 N.cm ${ }^{-2}$ and between the calf and the cushion around $1 \mathrm{~N} . \mathrm{cm}^{-2}$ ), a cluster of $98 \mathrm{~mm}^{3}$ and a maximum VM strain of $20.6 \%$ are recorded. The standard deviation for the VM strain is close to 0 showing that the differences in the calcaneus bone shape do not influence this factor. Nevertheless, the standard deviation of the cluster volume is $33 \mathrm{~mm}^{3}$ (33.8 ppt) showing that this factor (and the associated risks for pressure ulcers) is influenced by the calcaneus shape in this cushion configuration. Last, but not least, a cluster of $4,332 \mathrm{~mm}^{3}$ and a maximum VM strain of $50.6 \%$ are observed when the section below the heel is highly inflated while the rest of the cushion is soft (with an interface pressure between the heel and the cushion around $8 \mathrm{~N} . \mathrm{cm}^{-2}$ and between the calf and the cushion around $1 \mathrm{~N}^{-\mathrm{cm}^{-2}}$ ). The standard deviation for the cluster volume is $509 \mathrm{~mm}^{3}(11.8 \mathrm{ppt})$ while it is $3.0 \%$ for the VM strain $(6.0 \mathrm{ppt})$. It is important to note here that only 6 of the 18 patients reach the $50 \% \mathrm{VM}$ strain threshold limit. These results show again that the differences in the calcaneus bone 
shapes do influence the risk of pressure ulcer creation when the cushion is more inflated under the heel (case 5 in table 1). Furthermore, in this last case, the VM strain threshold of 50 $\%$ is exceeded and a cluster of $46 \mathrm{~mm}^{3}$ and a maximum VM strain of $52.5 \%$ are recorded.

Looking more into details, figure 5 plots the strains and " $20 \%$ clusters" volumes computed with the 18 calcaneus bones shapes. It is particularly interesting to note here that very significant differences can be observed in the maximal strains and corresponding volume values (for example, $31.3 \%$ and $2.31 \mathrm{~cm}^{3}$ for case \#1; $42.5 \%$ and $3.38 \mathrm{~mm}^{3}$ for case \#11, figure 6). This example clearly illustrates and quantifies the huge influence of the shape of the calcaneus bone on the computed internal strains values.

Finally, we note that in all those cases the maximum VM strain and the corresponding cluster are located at the interface between the fat and the calcaneus, figure 7 . When it is observed in the calf, this maximum is located at the interface between the fat and the muscle layer.

\section{Discussion}

The cluster volumes and VM strains presented in Table 1, figures 5 and 7, exhibit important variations between patients when the cushion is more inflated underneath the heel. Results obtained with the 18 models indeed highlight the paramount influence of the shape of the calcaneus bones on the volume of the largest cluster with strains above the $20 \%$ and $50 \%$ thresholds. We can conclude that the patient's individual morphology must be taken into account to assess the pressure ulceration risk level and no generic rule can be derived based on interface pressures alone. As concerns the $20 \%$ threshold, our models show high cluster 
volume variability: $327 \mathrm{~mm}^{3}$, which represents $12.1 \%$ of the total mean volume, when mildly inflated and $509 \mathrm{~mm}^{3}$, which represents $11.8 \%$ of the total mean volume, when highly inflated. The numerical experiments also indicate important variability of the maximum VM strains: $10.4 \mathrm{ppt}$ when mildly inflated and $6.0 \mathrm{ppt}$ when highly inflated, although the maximal strain measure alone should be considered with care given the many potential sources of local strain overestimation. This observation confirms the study made by Prof. Gefen ${ }^{8}$ on the above-mentioned analytical heel model. The results also suggest that both the part of the cushion being inflated as well as the magnitude of the interface pressures have an influence on the risk of pressure ulcer creation. Our results confirm the intuitive idea that a completely soft cushion should not create a pressure ulcer rapidly (but it could in the long run for some patients) while a cushion highly inflated below the back of the heel could create a pressure ulcer in about 10 minutes since a mean maximum VM strain of $52.5 \%$ (above the risk threshold of $50 \%$ provided by Loerakker et al. ${ }^{5}$ ) is recorded with a cluster volume of $46 \mathrm{~mm}^{3}$. However this volume represents only about five elements in our mesh and the interpretation of this result is therefore prone to uncertainty. To clarify the situation, the mesh would have to be locally refined and the cluster volume recomputed in order to enhance the solution accuracy - which was not done in this study. When the cushion has a section mildly inflated, below the calf or below the back of the heel, the apparition of a short term pressure ulcer seems unlikely but it is clear that it could happen in about 2 hours as it exceeds the $20 \%$ threshold provided by Loerakker et al. ${ }^{5}$, with a significant cluster volume of $223 \mathrm{~mm}^{3}$ and a mean maximum strain of $23.9 \%$ for the cushion mildly inflated below the calf, and a cluster volume of $2,698 \mathrm{~mm}^{3}$ and a mean maximum strain of $34.8 \%$ for the cushion mildly inflated below the heel. The cluster volumes mentioned above seem relevant given the refinement of 
the FE mesh as they contained more than 30 elements. This observation about the influence of the cushion corroborates the work by Tenenbaum et al. ${ }^{19}$, which used MRI to measure the "global" strains (i.e. strains computed by measuring the deformed/undeformed soft tissue height ratio between the calcaneus and the skin) of the heel soft tissues of 10 subjects for three different supports below the heel. The authors showed that heel padding devices have a significant effect on reducing the extent of deformations in the soft tissues, and that the features of their design have substantial influence on tissue deformations. Furthermore, the ranges of "global" strain values found in Tenenbaum et al. ${ }^{19}$ are similar to the "local" internal strains (VM strains measured at FE nodes) found in our study: between 20 and $60 \%$, depending on the type of padding. Our simulations also give the opportunity to evaluate the volume of tissue undergoing deformation, which could be used to determine the volume of tissues at risk of pressure ulcers.

The main limitation of our models concerns the choice of the Young moduli for the materials of the Neo Hookean constitutive law. We chose a Neo Hookean law to simulate realistically the quasi-incompressibility of the soft tissues and to model the large deformations due to the contact with the cushion. The choice of the mechanical parameters for each soft tissue layer is based on the literature (Sopher et al. ${ }^{8}$, Cheung et al. ${ }^{16}$ ) and could very well differ from a subject to another. We decided to use constant mechanical properties to avoid hindering the influence of the calcaneus bone geometry in this study. The subject-specific variation of the soft tissues properties would nonetheless need to be specifically studied in future work.

Another limitation of this work is the choice of a Neo Hookean material to simulate a cushion filled with compressed air (or fluid). This approximation was done to speed up the 
computation of the simulation by avoiding the integration of a fluid in the solver. To avoid modelling the air in the cushion as a fluid, we monitored the pressures at the interface between the cushion and the skin and we chose the material properties of the cushion in order to be lower than the maximal threshold recorded with the Zebris pressure sensor which constitutes a hard support generating a high pressure of $8 \mathrm{~N} . \mathrm{cm}^{2}$ under the heel for a $70 \mathrm{~kg}$ young healthy subject lying on his back. For a more accurate simulation, modeling the cushion as an envelope filled with air would be a better choice and the simulated cushion pressures could be used to drive the design of mattresses used in geriatric or reanimation units. In this study, the cushion pressure was not modelled and we rather relied on interface pressures which in our clinical experience cover most of real life situations. No simulation was done with a higher pressure under the Achilles tendon because the skin layer is very thin at this location, and that there is no fat to protect the tendon. As a consequence, a higher cushion pressure here would result immediately in high VM strains leading to higher risks of pressure ulcer creation.

\section{Conclusion}

The study presented in this article suggests that there is an influence of the calcaneus bone shape on the risk of pressure ulcer formation. It relies on FE modeling of the heel's soft tissues including the main biomechanical structures from the foot to the knee. The influence of 18 different shapes of calcaneus bones on the tissues deformation has been studied while the virtual leg was resting on a cushion with different levels of stiffness. The FE model of the heel is composed of four different soft tissue layers: namely the skin, fat, Achilles tendon, and 
muscles of the lower leg. Each of these layers follows a Neo Hookean constitutive law with different mechanical parameters. The bones of the lower leg are integrated in the model and coupled to soft tissues.

Results indicate that some shapes of the calcaneus bone induce higher risks for the development of posterior heel pressure ulcer. Two criteria were monitored during the FE simulations: (1) the volume of the largest cluster (with VM strains over $20 \%$ or $50 \%$ ) and (2) the maximum VM strains. The results are presented in table 1. The figures suggest that this influence is dependent of how the supporting cushion is inflated. The average deviations range from $11.8 \mathrm{ppt}$ to $68.4 \mathrm{ppt}$ for the cluster volume and from $2.9 \mathrm{ppt}$ to $10.4 \mathrm{ppt}$ for the mean maximum VM strain for the five different types of cushions. This indicates to which extent the calcaneus bone geometry has an influence on the risk of pressure ulcer creation since it is linked to the maximum strains in the soft tissues. This study additionally points out that various cushion pressures lead to different risks of pressure ulcer creation. A soft cushion does not seem to be a source of risk as concerns short term pressure ulcers (even though long term risk is present for 10 of the patients), while cushions uniformly mildly inflated or mildly inflated under one of the sections of the leg all lead to a risk of pressure ulcer creation in a time period around two hours (since the $20 \% \mathrm{VM}$ strain threshold is reached in all cases ${ }^{5}$ ). Even more important, this study shows that with a cushion highly inflated underneath the heel, there is a risk of short term pressure ulcer creation (since the $50 \% \mathrm{VM}$ strain threshold ${ }^{5}$ is reached for 6 of the patients).

The patient-specific biomechanical model of the lower leg presented here could provide insight on the behavior of soft tissues resting on cushions with different stiffness settings by estimating and localizing the higher strains inside the lower leg and on its surface. 
Consequently, when coupled to the output of a pressure sensor inserted between the lower leg and the cushion, our model could determine if pressure ulcers may or may not appear for a given subject. This modeling technique could be used to control a pneumatic prevention device for patients in reanimation or geriatric units.

Several issues need to be solved before achieving efficient pressure ulcer prevention. It seems that the location and shape of the bony structures are a key point in the process of pressure ulcer creation. Therefore, using a patient-specific model would be the first step to achieve to claim ulcer prevention. This will have two folds: modeling the anatomy of each patient, and integrating the individual's soft tissues mechanical properties. Finally, and most importantly, for a daily prevention of pressure ulcers, it is crucial to be able to compute the simulations presented above in a short time since Loerakker et al. ${ }^{5}$ introduced the fact that ulcers can be created in less than 10 minutes with strains over $50 \%$. With a computation time around 70 minutes, our model is far from reaching this goal. It will therefore be crucial to speed up the simulation time, either by investigating parallel computing, by reducing the number of elements or by pre-computing the simulations and using them as a library of possible cases.

\section{Acknowledgments}

Competing interests: Some authors are involved with the TexiSense Company (http://www.texisense.com/home_en).

Funding: This work is partly funded by the 2010 ANR TecSan IDS project, by the CAMI Labex (ANR-11-LABX-0004) and by the Institut Universitaire de France. 


\section{References}

1. Perneger T.V., C. Héliot, A.C. Raë, F. Borst, J.M. Gaspoz. Hospital acquired pressure ulcers. Risk factors and use of preventive devices. Arch Intern Med, 158 (17): 19401945, 1998.

2. Driver V.R., M. Fabbi, LA. Lavery, and G. Gibbons. The costs of diabetic foot: the economic case for the limb salvage team. J Am Podiatr Med Assoc. 100(5):335-41, 2010.

3. Reiber G.E. Epidemiology of Foot Ulcerations and Amputations in Diabetes, 6th ed., Mosby, 2010.

4. Mueller M.J. Etiology, evaluation, and treatment of the neuropathic foot. Crit Rev Phys Rehabil Med, 3:289-309, 1992.

5. Loerakker S., E. Manders, G.J. Strijkers, K. Nicolay, F.P.T. Baaijens, D.L. Bader, and C.W.J. Oomens. The effects of deformation, ischaemia and reperfusion on the development of muscle damage during prolonged loading. J. Appl. Phys. 111(4):1168$1177,2011$.

6. Armstrong D.G., and L.A. Lavery. Evidence-based options for off-loading diabetic wounds. Clin Podiatr Med Surg. 15:5-104, 1998.

7. Linder-Ganz E., N. Shabshin, Y. Itzchak, Z. Yizhar, I. Siev-Ner, and A. Gefen. Strains and stresses in sub-dermal tissues of the buttocks are greater in paraplegics than in healthy during sitting. J. Biomech. 41:567-580, 2008.

8. Gefen A. The biomechanics of heel ulcers. Journal of Tissue Viability. 19, 124-131, 2010 
9. Ledoux W.R., D.F. Meany, and H.J. Hillstrom. A quasi-linear, viscoelastic, structural model of the plantar soft tissue with frequency sensitive damping properties. $J$ Biomech Eng. 126:1-7, 2004.

10. Chen W.M., T. Lee, P. Vee-Sin Lee, and S.J. Lee. Effects of internal stress concentrations in plantar soft-tissue - preliminary three-dimensional finite element analysis. Med. Eng. \& Phys. 32:324-331, 2010.

11. Luboz V., A. Perrier, I. Stavness, J.E. Lloyd, M. Bucki, F. Cannard, B. Diot, N. Vuillerme, and Y. Payan. Foot Ulcer Prevention Using Biomechanical Modeling. Computer Methods in Biomechanics and Biomedical Engineering: Imaging \& Visualization (CMBBE: I\&V). 2014.

12. Sopher R., J. Nixon, E. Mc Ginnis, and A. Gefen. The influence of foot posture, support stiffness, heel pad loading and tissue mechanical properties on biomechanical factors associated with a risk of heel ulceration. Journal of the Mechanical Behavior of Biomedical Materials. 4, 572 - 582, 2011.

13. Lloyd J.E., I. Stavness, and S. Fels. Artisynth: a fast interactive biomechanical modeling toolkit combining multibody and finite element simulation. Soft Tissue Biomechanical Modeling for Computer Assisted Surgery, Studies in Mechanobiology, Tissue Engineering and Biomaterials, Vol.11. Springer, pp. 355-394, 2012.

14. Lobos C., Y. Payan, and N. Hitschfeld. Techniques for the generation of 3D Finite Element Meshes of human organs. Informatics in Oral Medicine: Advanced Techniques in Clinical and Diagnostic Tech. Hershey, PA: Medical Information Science Reference, pp. 126-158, 2010. 
15. Bonet J. \& R.D. Wood. Nonlinear Continuum Mechanics for Finite Element Analysis. Cambridge University Press, 2008.

16. Cheung J.T.M., M. Zhang, K.N. An. Effect of Achilles tendon loading on plantar fascia tension in the standing foot. Clinical Biomechanics, 21:194-203, 2006.

17. Harless E. The static moments of the component masses of the human body. Trans. of the Math-Phys., Royal Bavarian Acd. of Set., 8(1,2):09-96, 257-294, 1860.

18. Oomens C.W.J., O.F.J.T. Bressers, E.M.H. Bosboom, C.V.C. Bouten, and D.L. Bader. Can Loaded Interface Characteristics Influence Strain Distributions in Muscle Adjacent to Bony Prominences? Comput Methods Biomech Biomed Engin. 6(3):171-180, 2003.

19. Tenenbaum S., N. Shabshin, A. Levy, A. Herman, and A. Gefen. Effects of foot posture and heel padding devices on soft tissue deformations under the heel in supine position in males: MRI studies. Journal of Rehabilitation Research and Development. 50(8):1149-56, 2013. 
1

Figures and tables

2

3

4

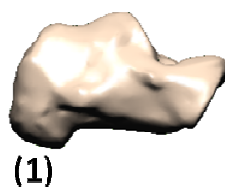

(1)

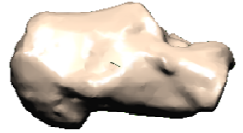

(7)

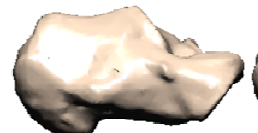

(13)

Figure $1 \mathrm{Luboz}$

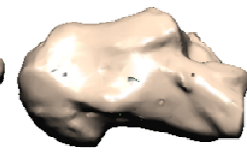

(2)

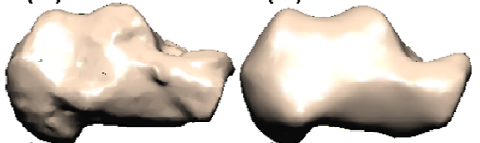

(8)

(9)

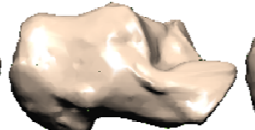

(14)

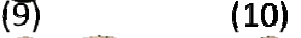

(15)
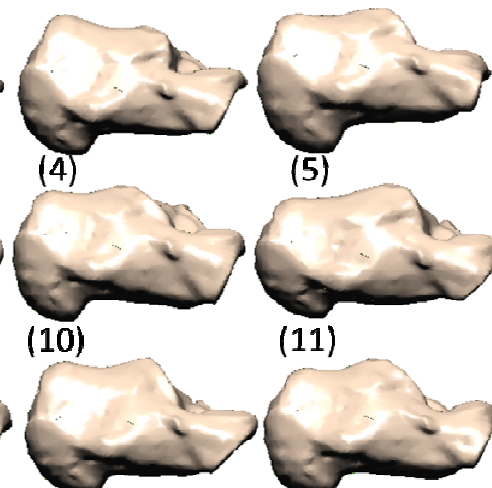

(4)

(5)

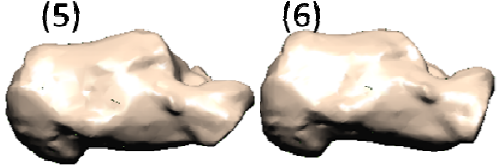

(11)

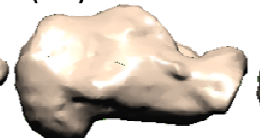

(17)
(12)
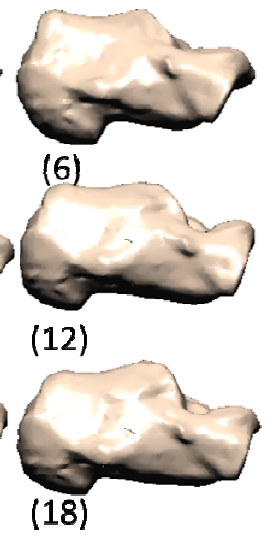

6 
7 Figure $2 \mathrm{Luboz}$
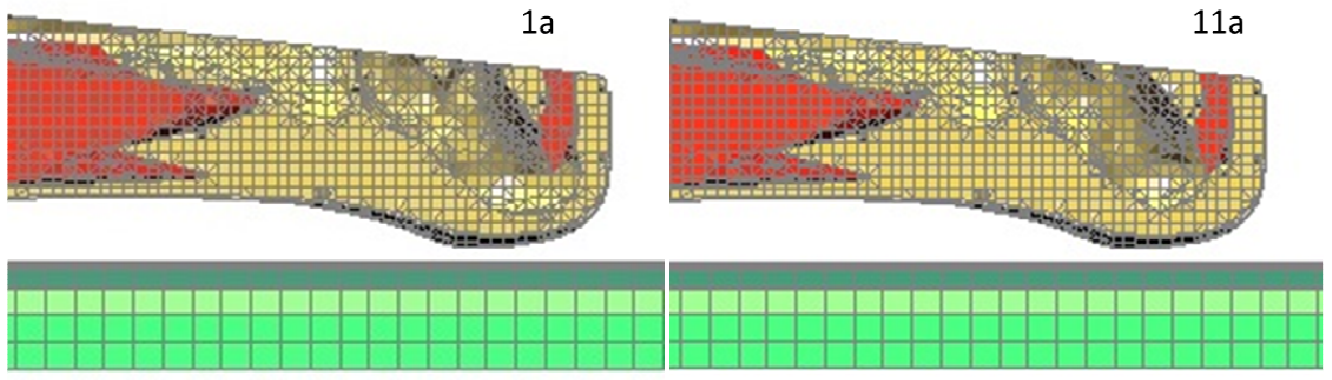

8

9 
Figure 3 Luboz

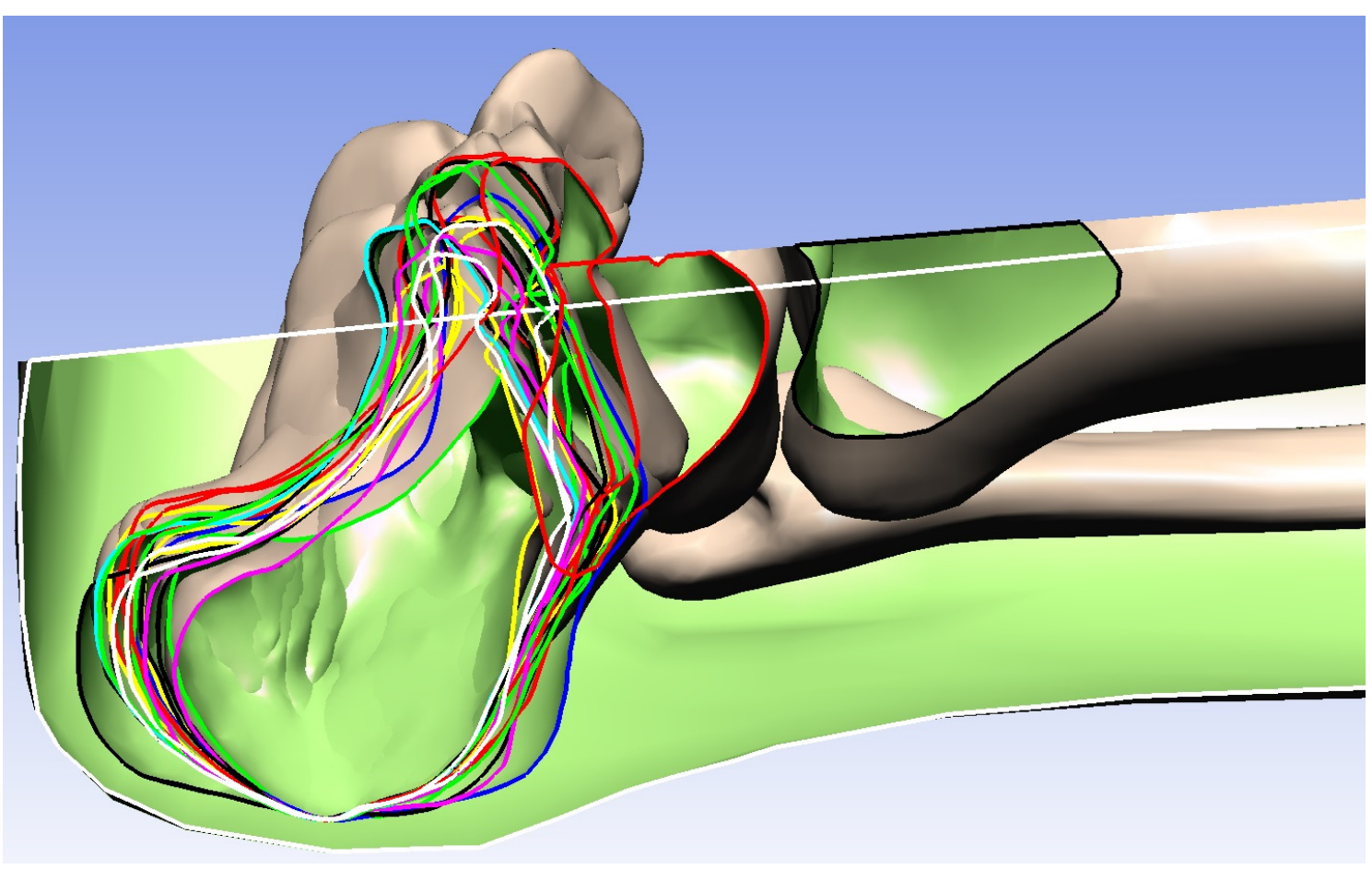

11 

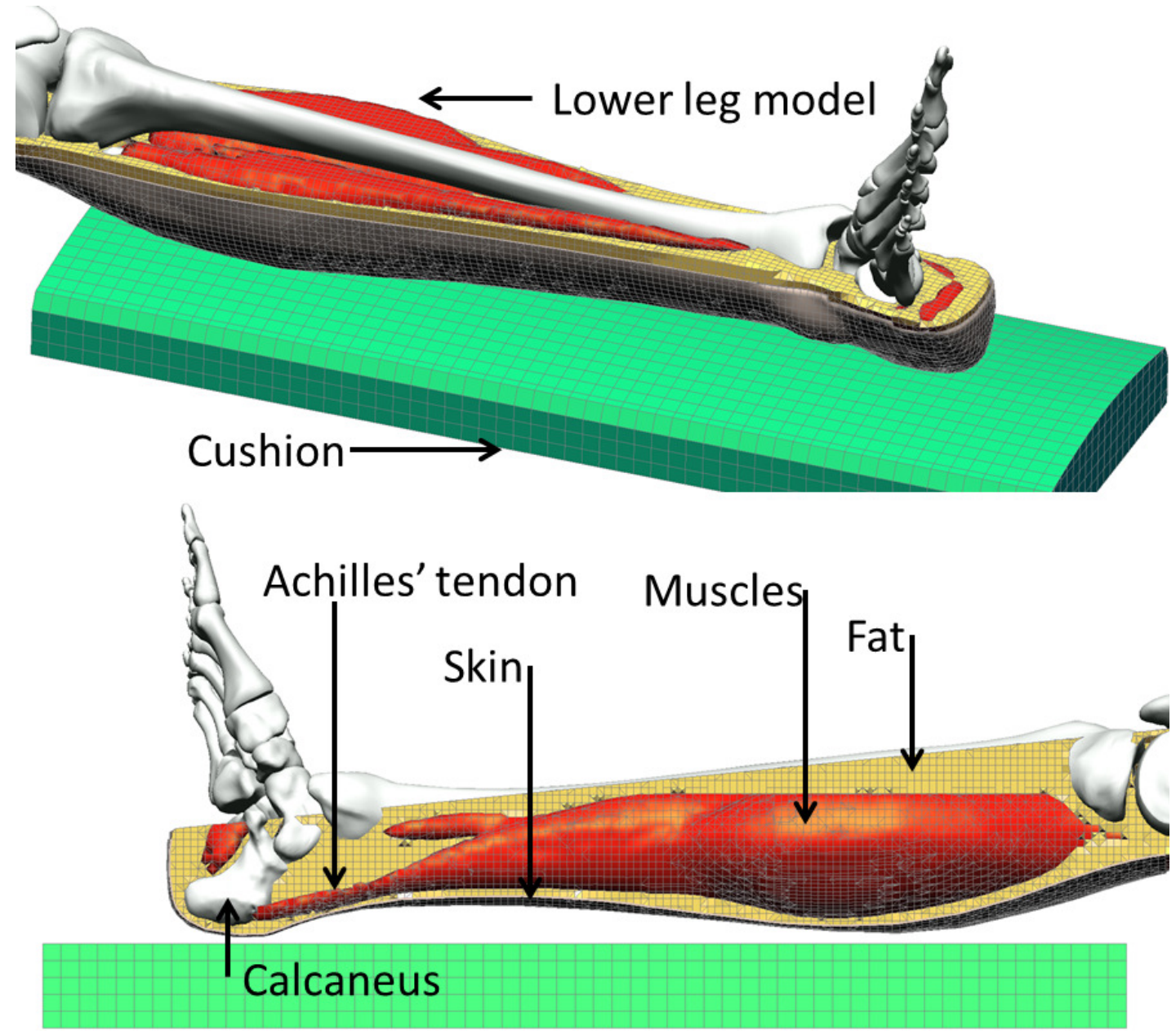
Figure 5 Luboz
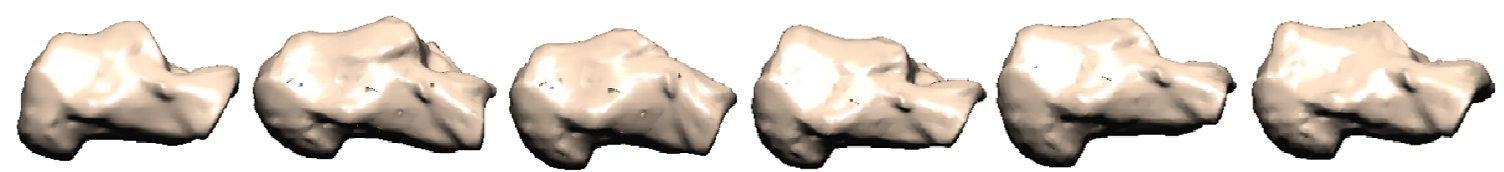

(1) $2.31 .3 \%$

(2) $\begin{aligned} & 31.1 \% \\ & 2.67 \mathrm{~cm}^{3}\end{aligned}$

(3) $2.89 \mathrm{~cm}^{3}$

(4) $2.2 \% \mathrm{~cm}^{3}$

(5) $2.78 \mathrm{~cm}^{3}$

(6) $33.5 \%$
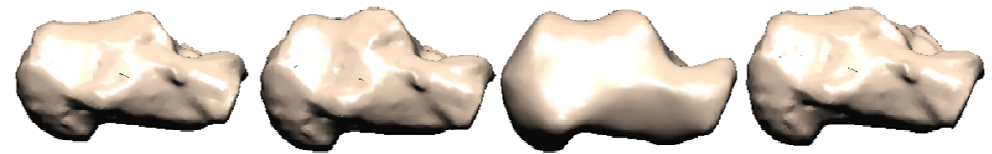

(7) $2.58 .7 \%$

(8) $34.4 \%$

(9) $\begin{aligned} & 30.7 \% \\ & 2.08 \mathrm{~cm}^{3}\end{aligned}$

$(10)^{32.4 \%} 2.93 \mathrm{~cm}^{3}$
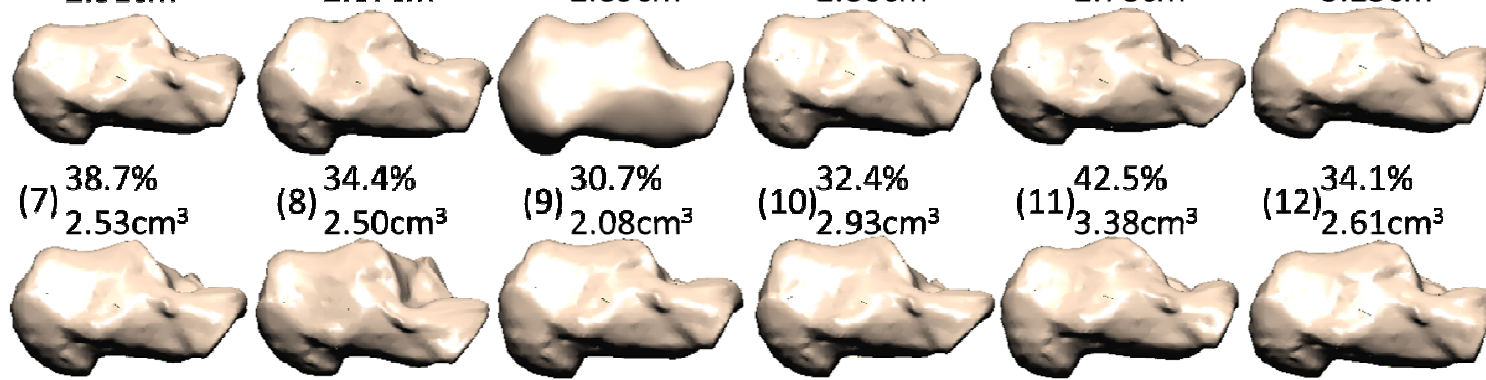

$(13)^{33.7 \%}$
$2.64 \mathrm{~cm}^{3}$

$(14)^{32.2 \%}$
$2.91 \mathrm{~cm}^{3}$
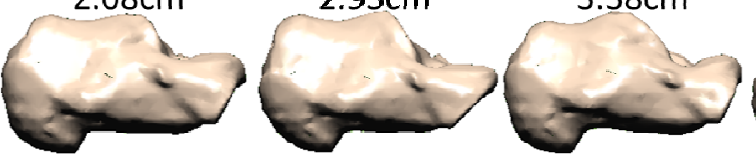

$(12)^{34.1 \%} 2.61 \mathrm{~cm}^{3}$

21

$(15)^{42.8 \%}$
$2.83 \mathrm{~cm}^{3}$

$(16)^{34.7 \%}$
$2.24 \mathrm{~cm}^{3}$

(17) $2.46 \mathrm{~cm}^{3}$

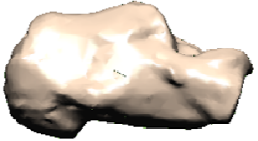

(18) $35.6 \%$

$2.75 \mathrm{~cm}^{3}$

22 
Figure 6 Luboz
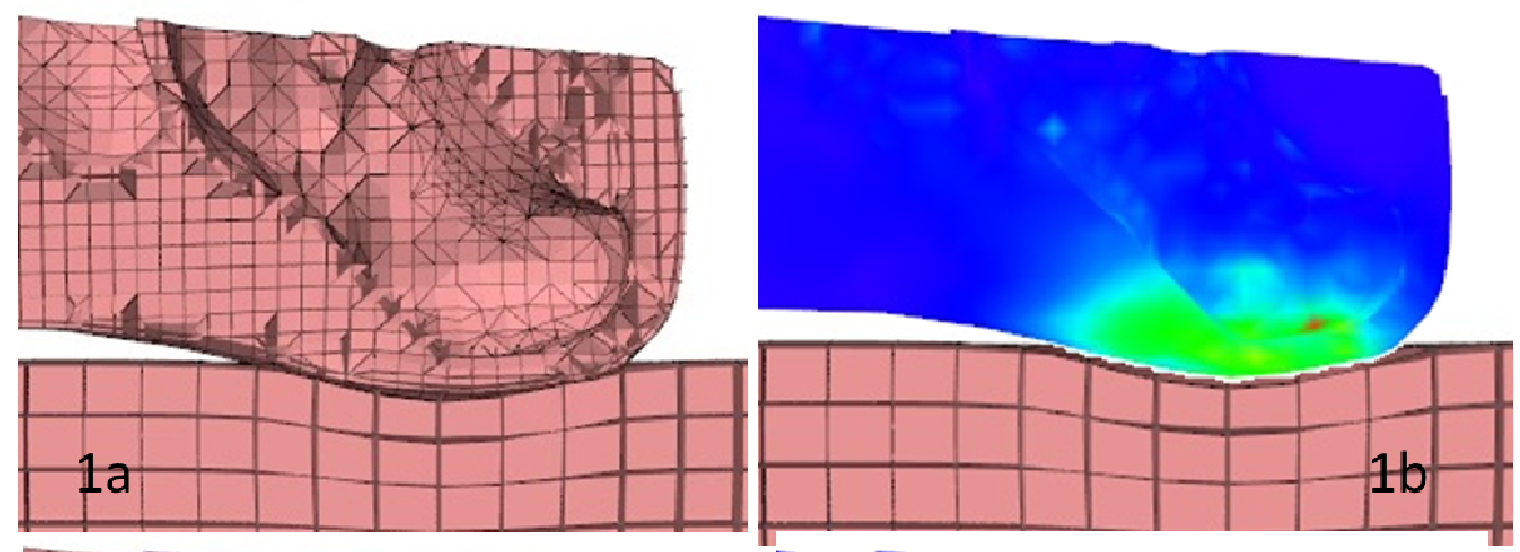

25
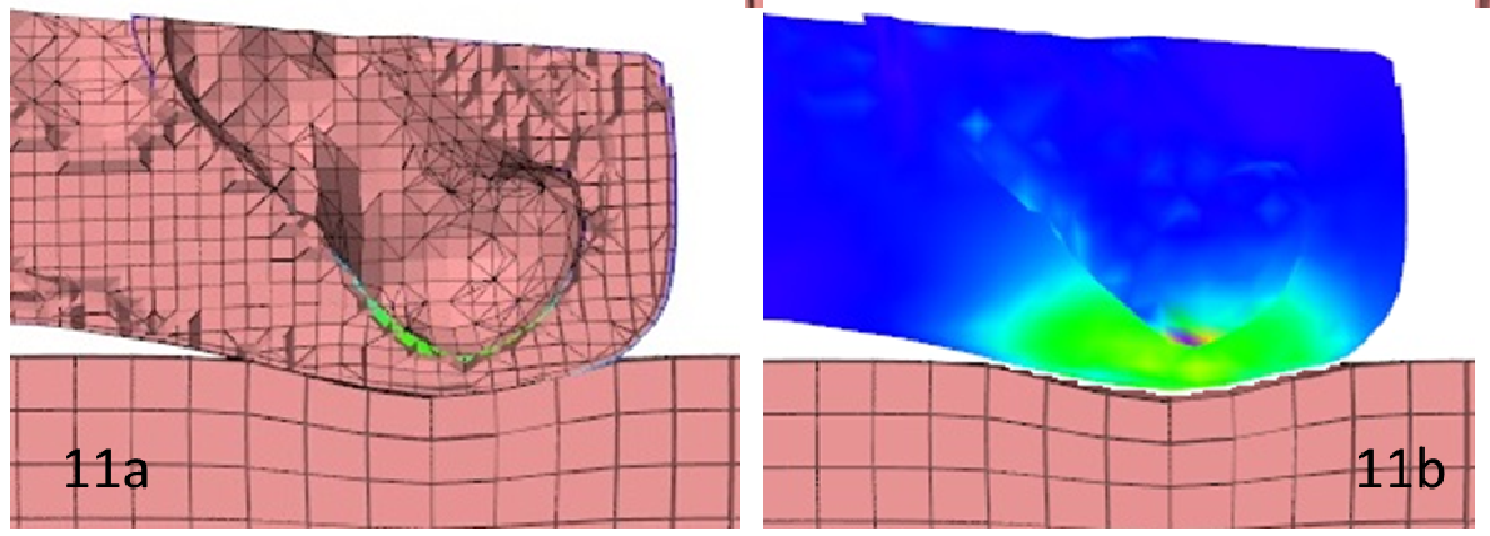

26 
27 Figure 7 Luboz

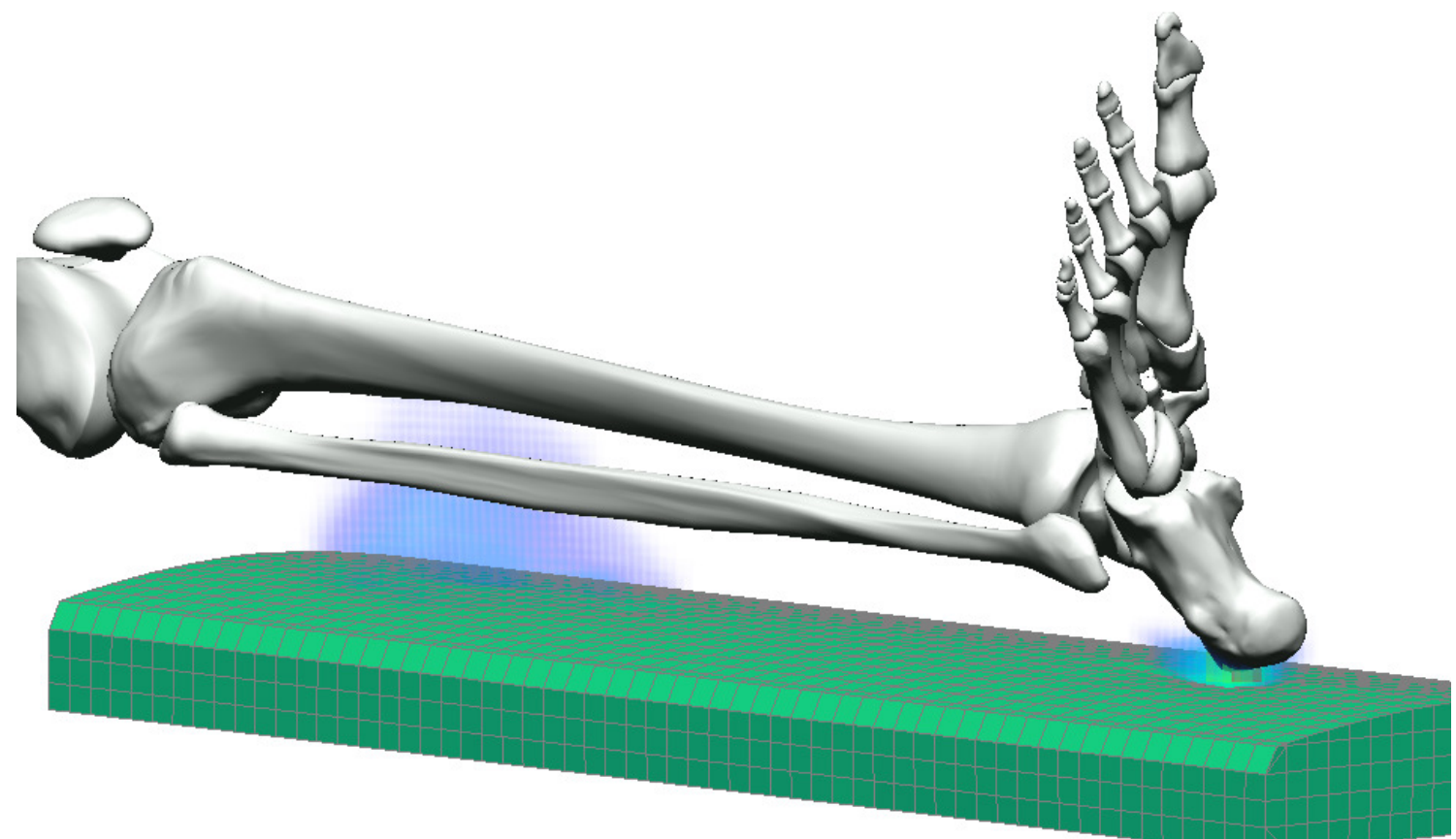

28

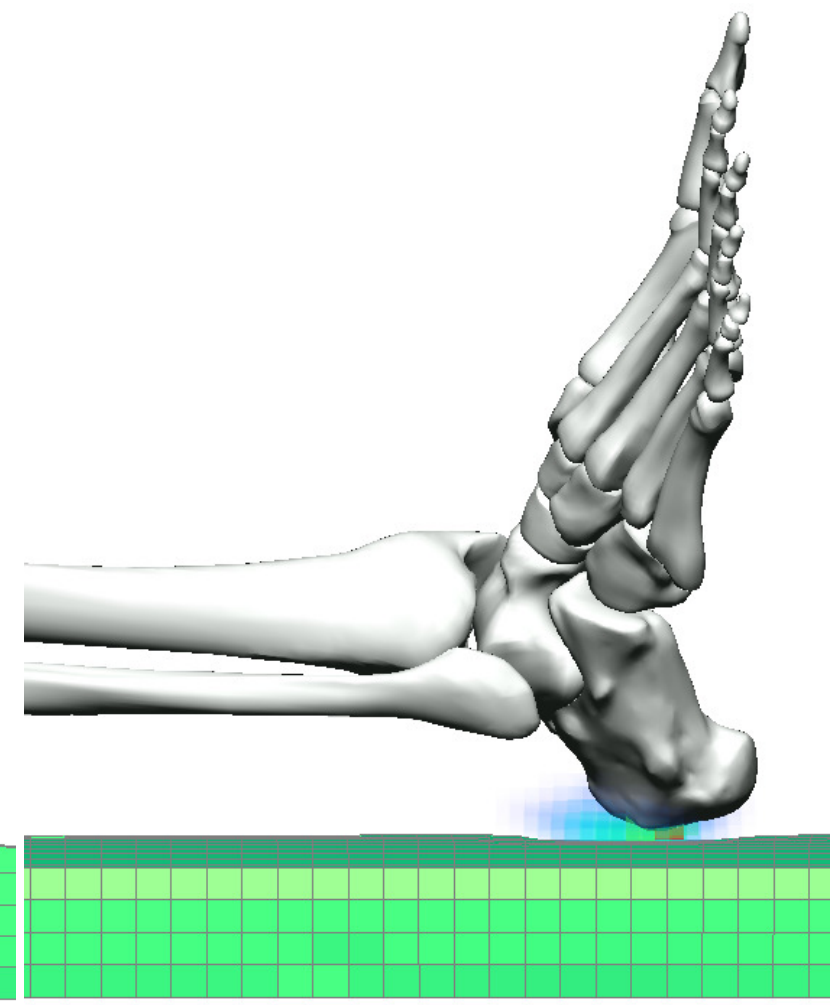




\begin{tabular}{|c|c|c|c|c|c|}
\hline Cushions' stiffness & $\begin{array}{l}\text { (1) All } \\
\text { soft }\end{array}$ & $\begin{array}{l}\text { (2) All soft } \\
\text { and calf mild } \\
\text { pressure }\end{array}$ & $\begin{array}{l}\text { (3) All soft } \\
\text { and heel mild } \\
\text { pressure }\end{array}$ & $\begin{array}{l}\text { (4) All } \\
\text { mild } \\
\text { pressure }\end{array}$ & $\begin{array}{l}\text { (5) All soft } \\
\text { and heel high } \\
\text { pressure }\end{array}$ \\
\hline $\begin{array}{l}\text { Mean volume (in } \mathrm{mm}^{3} \text { ) of } \\
\text { the maximum cluster with } \\
\text { a VM strain above } 20 \%\end{array}$ & 33 & 223 & 2,698 & 98 & 4,332 \\
\hline $\begin{array}{l}\text { Volume standard deviation } \\
\text { in } \mathrm{mm}^{3}\end{array}$ & 23 & 60 & 327 & 33 & 509 \\
\hline Deviation in \% & 68.4 & 26.9 & 12.1 & 33.8 & 11.8 \\
\hline $\begin{array}{l}\text { Mean maximum VM strain } \\
\text { in } \%\end{array}$ & $22.4 \%$ & $23.9 \%$ & $34.8 \%$ & $20.6 \%$ & $50.6 \%$ \\
\hline $\begin{array}{l}\text { Maximum VM strain } \\
\text { standard deviation in \% }\end{array}$ & $2.0 \%$ & $1.4 \%$ & $3.6 \%$ & $0.6 \%$ & $3.0 \%$ \\
\hline Deviation & 8.9 & 5,9 & 10.4 & 2.9 & 6.0 \\
\hline Only 10 subjects reach the 2 & $0 \%$ thresh & old & Only 6 subject\$ & reach the 5 & o\% threshold \\
\hline $\begin{array}{l}\text { Mean volume (in } \mathrm{mm}^{3} \text { ) of } \\
\text { the maximum cluster with } \\
\text { a VM strain above } 50 \%\end{array}$ & 0 & 0 & 0 & 0 & $\Gamma$ \\
\hline $\begin{array}{l}\text { Volume standard deviation } \\
\text { in } \mathrm{mm}^{3}\end{array}$ & 0 & 0 & 0 & 0 & 28 \\
\hline Deviation in \% & 0 & 0 & 0 & 0 & 59.8 \\
\hline $\begin{array}{l}\text { Mean maximum VM strain } \\
\text { in \% }\end{array}$ & 0 & 0 & 0 & 0 & $52.5 \%$ \\
\hline $\begin{array}{l}\text { Maximum VM strain } \\
\text { standard deviation in \% }\end{array}$ & 0 & 0 & 0 & 0 & 1.8 \\
\hline Deviation & 0 & 0 & 0 & 0 & 3.4 \\
\hline
\end{tabular}

31 
Captions:

Figure 1 - The 18 calcaneus bones extracted from CT scans and used to create the 18 different FE models

Figure 2- Example of two meshes, zoomed on the heel: 1a, for patient \#1's heel and 11a, for patient \#11's heel.

Figure 3 - Example of six of the 18 calcaneus bones artificially overlaid on the other bones' surfaces.

Figure 4 - Top: Finite element model of the lower leg, from heel to knee, lying on the cushion. To avoid having too many elements, the top part of the leg soft tissues are not included. Bottom: The four types of materials defining the lower leg FE model: skin (only one layer of elements around the leg, except above the tibia and near the knee), tendon, muscles (in red), and fat (in yellow). The bones are simulated by fixed nodes at their interfaces with the soft tissues.

Figure 5 - Strains and $20 \%$ cluster volumes computed for the 18 lower leg models, including different calcaneus bones shapes, for a cushion in case (3): soft pressures under the calf and Achilles' tendon, and a mild pressure under the heel.

Figure 6 - Example of two simulations with a cushion in case (3): soft pressures under the calf and Achilles' tendon, and a mild pressure under the heel. The top line shows the mesh for patient \#1's heel in its final state (1a), and the Von Mises strains in the final state (1b), with a maximum at $31.3 \%$. The bottom line shows the mesh for patient \#11's heel in its final state (11a), and the Von Mises strains in the final state (11b), with a maximum at $42.5 \%$. In both cases, the maximum Von Mises strain value of the scale (in red on the figure) is set to $45 \%$. 
Figure 7 - Cluster of the nodes with VM strains above $20 \%$ when the cushion is highly inflated below the heel and completely soft elsewhere. The maximum VM strain is located under the back of the heel, at the interface between the fat and the calcaneus bone. The VM strain goes from close to $0 \%$ (in blue) to $55.7 \%$ (in red).

Table 1 - Summary of the simulations for the 18 calcaneus shapes. For each of the VM strain thresholds of $20 \%$ and $50 \%$, the largest cluster volume below the heel, the standard deviation and the deviation in percentage points, the maximum VM strains below the heel, the standard deviation and the deviation in percentage points are averaged for five types of cushions: (1) completely soft, (2) completely soft except the calf section which is mildly inflated, (3), completely soft except the heel section which is mildly inflated, (4) uniformly mildly inflated, and (5) completely soft except the heel section which is highly inflated. Note that the figures given for the first column is an average of only 10 of the 18 patients as only 10 of them reach the $20 \%$ threshold. Similarly, the bottom of the last column is an average of only 6 of the 18 patients as only 6 of them reach the $50 \%$ threshold. 